\title{
Modification of the Energy Spectrum of UHECR by the Galactic Magnetic Field for Anisotropic Arrival Directions
}

\section{Björn Eichmann*}

Ruhr Astroparticle and Plasma Physics Center (RAPP Center), Ruhr-Universität Bochum, Institut für Theoretische Physik IV, 44780 Bochum, Germany

E-mail: eiche@tp4.rub. de

\section{Tobias Winchen}

Astrophysical Institute, Vrije Universiteit Brussel, Pleinlaan 2, 1050 Brussels, Belgium Now at Max-Planck-Institut für Radioastronomie (MPIfR), Auf dem Hügel 69, 53121 Bonn, Germany

E-mail: tobias.winchenerwth-aachen.de

\begin{abstract}
Recently, the Pierre Auger Observatory observed a large scale anisotropy in the arrival direction of Ultra-High-Energy Cosmic Rays (UHECRs). As the Galactic magnetic field cannot produce such an anisotropy, the distribution of UHECRs hitting the Milky Way has to be anisotropic already. In this case, the Galactic magnetic field may change the observed energy spectrum with regard to the spectrum outside of the Milky Way as the UHECR intensity from certain directions can be enhanced and suppressed, respectively. We discuss the effect for the case of a dipole anisotropy and a commonly used magnetic field model, and demonstrate that the effect is negligible if the anisotropy outside the Milky Way is small, i.e. a dipole amplitude of about a few percentage.
\end{abstract}

36th International Cosmic Ray Conference -ICRC2019-

July 24th - August 1st, 2019

Madison, WI, U.S.A.

\footnotetext{
* Speaker.
} 


\section{Introduction}

Ultra-High-Energy Cosmic Rays (UHECRs) are composed of fully ionized nuclei, so that their distribution cannot be decoupled from the influence of magnetic fields. However, there is a popular, limiting scenario - derived from the so-called Liouville theorem - which states that the CR distribution at Earth has to be isotropic if it is isotropic outside of the Milky Way. Hence, magnetic fields cannot introduce anisotropies in the first place and also the energy spectrum is conserved if the flux is isotropic outside the Milky Way.

But the Pierre Auger Observatory $[3,1]$ recently reported a large-scale dipole anisotropy with an amplitude of $\approx 6.5 \%$ at energies $>8 \mathrm{EeV}$ that increases with energy. Further indications of a strong anisotropy at Earth are given by the observed differences of the UHECR intensity at about $60 \mathrm{EeV}[2]$ between the northern and the southern hemisphere.

Thus, the UHECRs that enter the Milky Way are most likely anisotropically distributed, so that the intensity of UHECRs gets additionally modified by the propagation through the Galactic magnetic field. So, how much does the energy spectrum at Earth tell us about the energy spectrum outside the Milky Way or vice versa how much is the energy spectrum at Earth changed by the fact of an anisotropic UHECR distribution outside our Galaxy?

Here, we investigate this question by using the publicly available code CRPropa3 [4]. First, in section 2 we introduce the theoretical background and the used simulation techniques, before our approach and the corresponding results are shown in section 3 .

\section{Method}

Based on Liouville's theorem an efficient, commonly used CR propagation technique has been developed: the so-called backtracking method, where anti-particles are propagated backwards to obtain the trajectories of the regular particles that hit the observer.

But this theorem only holds under certain requirements. First, the magnetic field needs to be in a stationary condition, and secondly, the particles are not allowed to interact with each other. Thus, the charged particles predominantly suffer from the Lorentz force and the system satisfies the Hamiltonian equations, so that the phase space density $f=\mathrm{d} N / \mathrm{d} \mathbf{x} \mathrm{d} \mathbf{p}$ is conserved, i.e. $\mathrm{d} f / \mathrm{d} t=0$. Hence, the density along the dynamical trajectory needs to be conserved. In the following, we suppose an ideal detector that counts the number of particles per unit time $\mathrm{d} t$, per unit energy $\mathrm{d} E$, per unit area $\mathrm{d} A=r^{2} \mathrm{~d} \Omega$, per unit solid angle $\mathrm{d} \Omega$, so that the particle intensity resulting from $f$ can be defined as

$$
I=\frac{f(\mathbf{x}, \mathbf{p}) \mathrm{d} \mathbf{x} \mathrm{d} \mathbf{p}}{\mathrm{d} t \mathrm{~d} E \mathrm{~d} A \mathrm{~d} \Omega}=f(\mathbf{x}, \mathbf{p}) p_{r}^{2} \frac{\mathrm{d} r}{\mathrm{~d} t} \frac{\mathrm{d} p_{r}}{\mathrm{~d} E}=f(\mathbf{x}, \mathbf{p}) p_{r}^{2},
$$

where $\mathrm{d} E / \mathrm{d} p_{r}=p_{r} /(\gamma m)=\mathrm{d} r / \mathrm{d} t$ has been used in the last step. So, also the intensity $I$ stays constant on the particle's trajectory according to Liouville's theorem and hence, the intensity in all allowed directions at any point is the same, if the particles are homogeneously and isotropically distributed at infinity, as already noted by Lemaitre and Vallarta [8]. The backtracking approach provides the allowed directions as those where the corresponding trajectory ends up at the outer sphere, e.g. the edge of our Galaxy. For the Galactic lens [5, 4], that is used in this work, $100 \times$ 50,000 particles were backtracked for each of 176 logarithmically binned energies from $0.1 \mathrm{EeV}$ 
to $300 \mathrm{EeV}$. Hereby, the Galactic magnetic field model from Jansson and Farrar [6] - hereafter referred to as JF12 - is used. For all trajectories the directions on Earth and the edge of the Galaxy were binned in $\sim 50000$ bins using Healpix to obtain a matrix that can be used to transform the flux distribution outside the Galaxy to an observed distribution on Earth. Thus, a homogeneous and isotropic CR intensity outside the Milky Way necessarily yields also an isotropic, constant intensity at Earth. Note that this only holds for an unidirectional detector with an infinitesimal cross-section. Further, this method cannot account for stochastic energy losses, due to its irreversibility. However, the propagation distance of UHECRs through the Galactic magnetic field is typically small to extragalactic distances of more than some tens of Mpc, so that also the occurring energy losses within the Galaxy are negligible.

To determine the change of intensity due to a given dipole anisotropy at the edge of the Milky Way, we first build an ideal dipole distribution with a dipole $\vec{d}=d \vec{e}_{d}$ of a certain strength $d$ and a direction $\vec{e}_{d}$. In doing so, an isotropic map $M_{\mathrm{iso}}(\vec{e})$ that is given by an isotropic distribution of unit vectors $\vec{e}$ is modified by the scalar product of the isotropic map vectors with the dipole, so that the dipole distribution is given by $M_{\mathrm{d}}=M_{\mathrm{iso}}(\vec{e}) \cdot \vec{d}$. Thus, the intensity $I_{\mathrm{d}}$ of UHECRs at the edge of the Milky Way is given by the sum of its entries. Using the Galactic lens that has been derived from the JF12 model, the UHECR distribution at Earth $M_{\mathrm{obs}}$ can be determined from the given dipole map. Due to the strong impact by the regular components of this Galactic magnetic field model the resulting distribution $M_{\mathrm{obs}}$ depends very much on $d$ and $\vec{e}_{d}$. However, we are only interested in the resulting UHECR intensity $I_{\mathrm{obs}}$ at Earth, which is again given by the sum of the entries of $M_{\text {obs }}$. Thus, the intensity modification at a given energy is given by $I_{\mathrm{obs}} / I_{\mathrm{d}}-1$. We suppose that the dipole anisotropy at the edge of our Galaxy is similar to the observed dipole anisotropy at Earth, so that $0.01 \lesssim d \lesssim 0.1$ between 4 and $30 \mathrm{EeV}$. Moreover, $d$ likely shows a rather high value at high energies and a small value at low energies, similar to the observations at Earth [1].

\section{Results}

The Fig. 1 shows that the resulting modification of the UHECR intensity is only at the level of a few percentage at most with a strong decrease at energies $>10 \mathrm{EeV}$. Independent of $d$ the modification of the intensity even vanishes for certain directions $\vec{e}_{d}$. On the other hand, a dipole anisotropy towards the Galactic center $\left(l \simeq 0^{\circ}\right)$ reduces the UHECR intensity at Earth — in particular close to $b=-30^{\circ}$, whereas a dipole in the opposite direction $\left(l \simeq 180^{\circ}\right)$ amplifies the intensity at Earth. The magnetic fields are the strongest close to the Galactic center. Thus, the UHECR intensity is reduced the most if the majority of them reaches the Milky Way from this direction, as they likely get deflected in the central region of the Galaxy.

Supposing a power law behavior of the UHECR intensity $I_{\mathrm{obs}}(E)=I_{0} E^{-\gamma}$ between $E_{0}$ and $E_{1}$ at Earth, we estimate the change of the spectral index as

$$
\Delta \gamma \simeq-\frac{\log \left(I_{\mathrm{obs}}\left(E_{0}\right) / I_{\mathrm{obs}}\left(E_{1}\right)\right)}{\log \left(E_{0} / E_{1}\right)} .
$$

Note that $\Delta \gamma$ depends on the chosen energies of reference, as shown in Fig. 2. But even at small energies where the intensity modification changes the most with energy, e.g. for $E_{0} \simeq 5 \mathrm{EeV}$ and $E_{1} \simeq 10 \mathrm{EeV}$, the change of the spectral index yields $\Delta \gamma \lesssim 0.04$ for $d \lesssim 0.1$. In addition, we show 

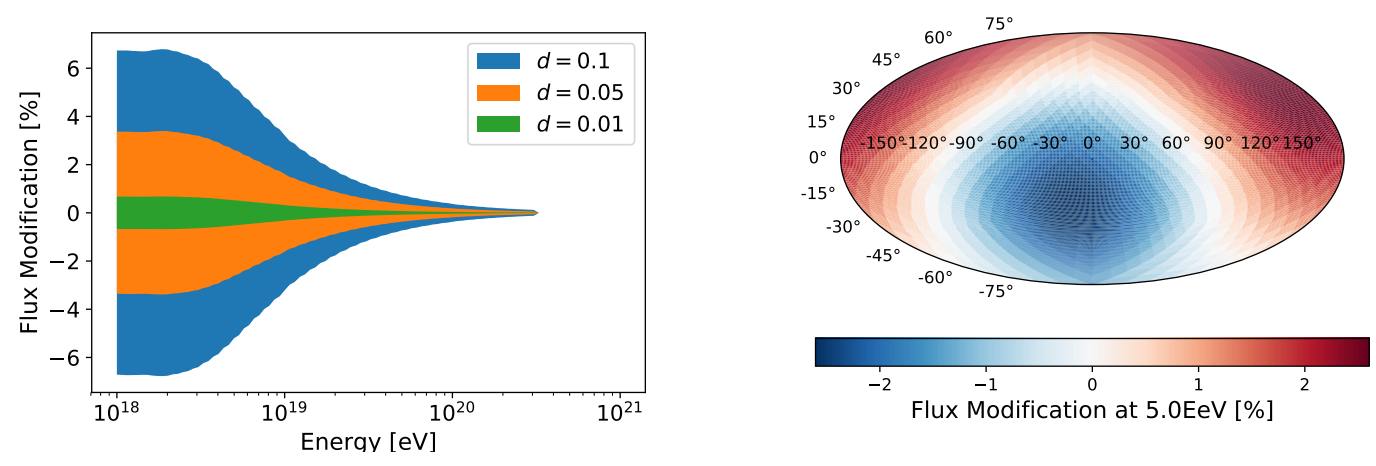

Figure 1: Left: The range of the intensity modification dependent on the energy for three different dipole strengths. Right: The intensity modification of UHECRs with $5 \mathrm{EeV}$ dependent on the Galactic coordinates of the dipole direction for a dipole amplitude of 5\%. Note that the Auger collaboration reported [1] a dipole with an amplitude of $2.5 \%$ and a direction of $(l, b)=(287,-32)$ at Earth for the given median of the energy.
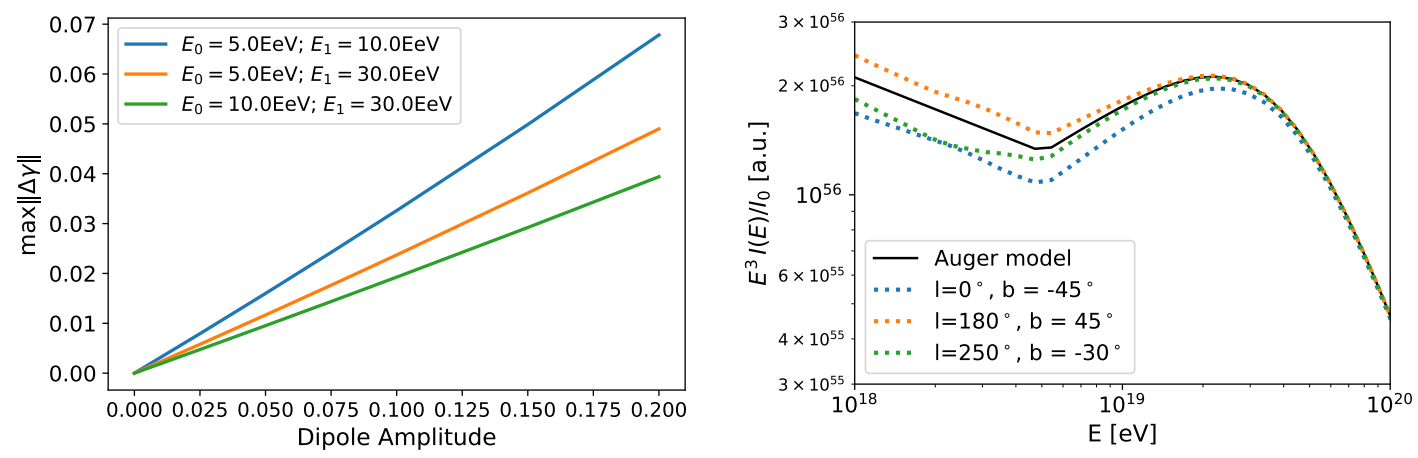

Figure 2: Left: The maximal change of the spectral index dependent on the dipole strength for three different sets of reference energies. Right: The resulting change of the UHECR spectrum in the extreme case of a dipole amplitude of 50\% at the edge of the Milky Way for three different dipole directions. The black line refers to the suggested UHECR spectrum from observations [7], that in principle keeps its shape outside the Milky Way in the case of a vanishing anisotropy.

that for a dipole amplitude that is about an order of magnitude higher outside the Milky Way, we obtain a significant change of the UHECR intensity at energies $\lesssim 30 \mathrm{EeV}$ if the dipole direction is towards the Galactic center $\left(l=0^{\circ}\right)$ or towards its anti-center $\left(l= \pm 180^{\circ}\right)$ as already indicated by the right Fig. 1 . For a dipole direction with $(l, b)=\left(250^{\circ},-30^{\circ}\right)$ which is about the observed dipole directions at Earth, we obtain even for a dipole amplitude of 50\% no significant effect on the UHECR spectrum above $5 \mathrm{EeV}$. However, the spectrum shows a slightly different spectral behavior at lower energies due to the modification by the Galactic magnetic field. Though the Fig. 3 exposes that the JF12 field is only able to reduce the dipole amplitude by an order of magnitude in the case of UHECRs with an energy $\lesssim 1 \mathrm{EeV}$ that arrive at the Milky Way from $l=0^{\circ}$ or $l=180^{\circ}$. Hereby, the relative change $\left(d_{\mathrm{obs}}-d\right) / d$ of the dipole strength is independent of $d$. 

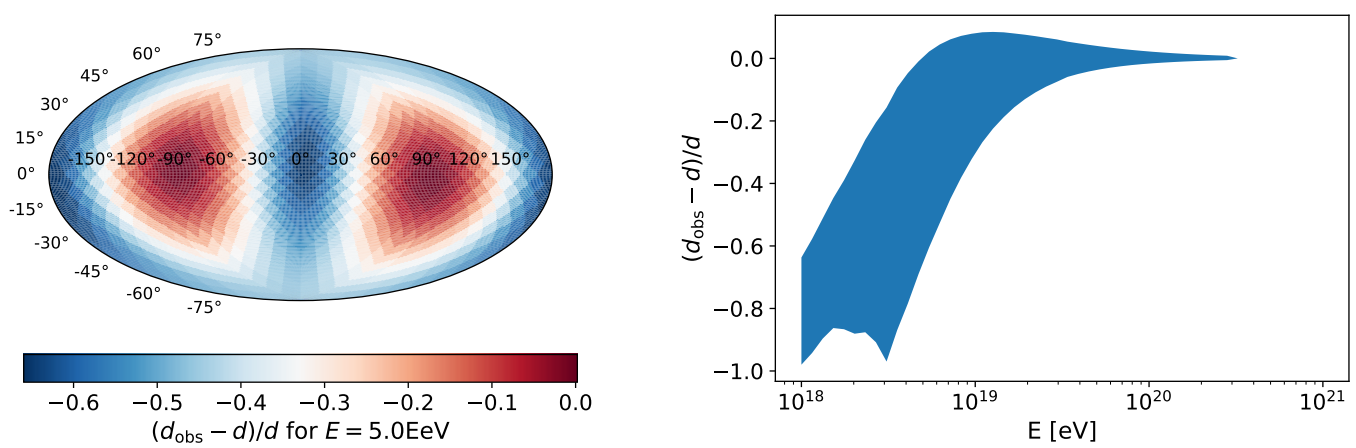

Figure 3: Left: The relative change of the dipol strength dependent on the initial dipole direction for a CR energy of $5 \mathrm{EeV}$. Right: The possible range of the relative change of the dipol strength dependent on the CR energy.

\section{Conclusions}

In total, we have shown that a dipole amplitude up to $10 \%$ has an impact on the resulting UHECR intensity that is at most at the order of the uncertainty of the observations. Hence, even if future observations show significant dipole anisotropies of the UHECRs at smaller energy regimes, the UHECR intensity at Earth will not significantly differ from the UHECR intensity outside our Galaxy. Here we assumed a pure proton composition - in the realistic case of a heavier composition the effect will be slighlty larger. However, the common assumption of the conservation of CR intensity from the outside to the inside is also appropriate in the case of the recently observed small dipole anisotropies. And even in the case of a significantly higher dipole strengths outside the Milky Way, the observed directions of the dipole indicate that the modification of the UHECR intensity above $5 \mathrm{EeV}$ stays negligible, if the dipole direction is not changed significantly by the Galactic magnetic field. However, strong anisotropies outside the Milky Way can in principle cause a completely different spectral behavior of the UHECRs at Earth, that might even explain some of the observed spectral kinks. But, the JF12 magnetic field is not strong enough to reduce the necessary strength of the dipole anisotropy outside the Milky Way to the observed anisotropy level at Earth at CR energies $\gtrsim 5 \mathrm{EeV}$. But note that multipole moments of higher order can have a significant different impact than the considered dipole anisotropy.

\section{References}

[1] A. Aab et al. "Large-scale Cosmic-Ray Anisotropies above $4 \mathrm{EeV}$ Measured by the Pierre Auger Observatory". In: ApJ 868.1 (2018), p. 4.

[2] R. U. Abbasi et al. "Evidence for Declination Dependence of Ultrahigh Energy Cosmic Ray Spectrum in the Northern Hemisphere”. In: arXiv:1801.07820 (2018).

[3] A. Aab et al. "Observation of a Large-scale Anisotropy in the Arrival Directions of Cosmic Rays above $8 \times 10^{18}$ eV”. In: Science 357.6537 (2017), pp. 1266-1270.

[4] R Alves Batista et al. "CRPropa 3 - a Public Astrophysical Simulation Framework for Propagating Extraterrestrial Ultra-High Energy Particles”. In: JCAP 1605 (2016), p. 038. 
[5] H.-P. Bretz et al. "PARSEC: A parametrized simulation engine for ultra-high energy cosmic ray protons". In: Astropart. Phys. 54 (2014), pp. 110-117.

[6] R. Jansson and G. R. Farrar. "A New Model of the Galactic Magnetic Field”. In: Astrophys. J. 757 (2012), p. 14.

[7] J. Abraham et al. "Measurement of the Energy Spectrum of Cosmic Rays above $10^{18} \mathrm{eV}$ using the Pierre Auger Observatory". In: Phys. Lett. B 685 (2010), pp. 239-246.

[8] G. Lemaitre and M. S. Vallarta. "On Compton's Latitude Effect of Cosmic Radiation". In: Phys. Rev. 43 (2 1933), pp. 87-91. 